ISSN 1981-416X

Licenciado sob uma Licença Creative Commons

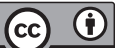

\title{
Didactique professionnelle (DP) et la théorie des situations didactiques (TSD): une perspective de complémentarité au Brésil
}

\section{Didática profissional (DP) e a teoria das situações didáticas (TSD): uma perspectiva de complementaridade no Brasil}

Professional Didactics (PD) and the Didactical Situations Theory (DST): a perspective of complementarity in Brazil

\section{Francisco Regis Vieira Alves*}

\section{Resumée}

Le présent travail présente une proposition théorique, fortement influencée par les domaines de recherche française de la didactique professionnelle (DP) et aussi la didactique des mathématiques (DM), en développement au Brésil. Ainsi, avec une plus grande attention adressée au professeur de mathématiques, en formation initiale et continue, le but est de déterminer un caractère de complémentarité et d'application des deux champs théoriques visant à mieux comprendre et modéliser l'activité et les éléments régulateurs de l'activité professionnelle de l'enseignant. En outre, il est souligné l'utilisation de la technologie afin de comprendre des certaines notions fondamentales pour la (DP) et la (DM).

Mots-clés: Didactique professionnelle. Théorie de la situation. Professeur de mathématiques.

"FRVA: Doutor em Educação, e-mail: fregis@ifce.edu.br 


\section{Resumo}

O presente trabalho apresenta uma proposta teórica fortemente influenciada pelos campos de pesquisa franceses de didática profissional (DP) e, também, da didática da matemática (DM), em desenvolvimento no Brasil. Assim, com uma maior atenção ao professor de matemática, na formação inicial e continuada, o objetivo é determinar uma complementaridade e aplicação dos dois campos teóricos visando uma melhor compreensão e modelagem da atividade e dos elementos regulatórios da aprendizagem e da atividade profissional do professor. Além disso, enfatiza-se o uso da tecnologia para entender alguns conceitos básicos para (DP) e a (DM).

Palavras-chave: Didática Profissional. Teoria das Situações. Professor de Matemática.

\section{Abstract}

The present work presents a theoretical proposal strongly influenced by the French research fields of professional didactics (DP) and also of didactics of mathematics (DM), in development in Brazil. Thus, with a greater attention to the mathematics teacher, in the initial and continued formation, the objective is to determine a complementarity and application of the two theoretical fields aiming at a better understanding and modeling of the activity and the regulatory elements of learning and the professional activity of the teacher. In addition, the use of technology is emphasized to understand some basic concepts for (DP) and (DM).

Keywords: Professional Didactics. Situation Theory. Professor of Mathematics. 


\section{Introduction}

Au Brésil, ily a une influence importante et une tradition d'études dans le domaine de la didactique des mathématiques (DM), qui traduit d'une attention particulière portée sur les phénomènes d'enseignement et d'apprentissage, et notamment sur les interactions entre les éléments du triangle didactique classique, dont les sommets sont représentés dans la littérature par: élèves - enseignant - savoir. Ainsi, pendant une vingtaine d'années, nous rencontrons plusieurs travaux de recherches développés au Brésil, qui nous permettent de souligner des contributions importantes de recherches centrées sur la théorie des situations didactiques (TSD). Ces travaux ont tendance à essayer de comprendre des phénomènes intrinsèques de l'enseignement et de l'apprentissage des mathématiques, c'est-à-dire, qu'ils analysent les processus de transmission et d'acquisition de différents contenus d'une discipline, en particulier, en s'associant aux spécificités de ce contenu et l'activité des élèves en classe.

Par ailleurs, puisqu'il est question d'un cadre théorique au Brésil qui s'approprie des phénomènes expérimentaux, en particulier des phénomènes issus du fonctionnement réel de la classe, on espère qu'au fil du temps, certains éléments, l'hypothèses théoriques et conceptions transmis par la théorie des situations didactiques (TSD) s'améliorent et même se généralisent. De plus, nous décrirons quelques notions qui, progressivement, ont acquis un emploi récurrent dans les études et recherches développées au Brésil (ALVES, 2018a).

Nous avons remarqué également certaines déficiences découlant de la nécessité de comprendre le rôle dialectique et pragmatique de l'enseignant des Mathématiques, qui ne peut être déterminé seulement par le champ épistémique d'une connaissances scientifiques ou même par les facteurs de conditionnement de la classe, ce qui a conduit à l'introduction de nouveaux cadres théoriques pour appuyer ces nouvelles recherches.

Ainsi, nous croyons qu'il est nécessaire de prendre en compte le rôle de l'enseignant, en particulier, en essayant de lui donner des moyens pour construire ses propres séquences de situations didactiques 
et situations didactiques professionnelles (SDP) de façon à rendre plus efficace l'enseignement des Mathématiques. Ceci nous a conduit à essayer de trouver des moyens qui puissent aider les enseignants à acquérir progressivement des compétences professionnelles au sein de leur propre institution de travail, face aux tâches quotidiennes et aux caractéristiques de la profession. Ainsi, nous allons commencer la section suivante a partir de la prise en compte de certains éléments de la didactique des mathématiques (DM), avec une forte influence dans la recherche française.

\section{La Didactique des Mathématiques (DM)}

Après avoir observé l'influence des recherches françaises en didactique des mathématiques (DM) et l'insertion encore faible des recherches françaises en didactique professionnelle (DP) ici au Brésil, il nous semble important de clarifier quelques points qui peuvent nous aider à mieux développer nos propres recherches d'une manière plus systématique.

Il convient tout d'abord de remarquer qu'en général nous nous plaçons dans un cadre théorique qui s'approprie des phénomènes expérimentaux, en particulier des phénomènes issus du fonctionnement réel de la classe, provenant d'un paradigme européen fortement épistémique et didactique. C'est dans cette perspective Alves (2016; 2017; 2018a; 2018b) développe ses recherches, en s'appuyant sur des notions de didactique des mathématiques (DM) déjà récurrentes dans les recherches brésiliennes.

D'abord, il nous semble important de souligner qu'en 2016, le premier congrès international sur la didactique des mathématiques a eu lieu au Brésil. À ce moment, Perrin-Glorian et Bellemain (2016) ont présentéune étude historique sur l'évolution de la didactique des mathématiques, dont nous pouvons remarquer certains éléments dans l'extrait qui suit:

La didactique des Mathématiques est née en France dans le contexte de la rénovation de l'enseignement de la fin des années 60 et du 
début des années 70, a partir de l'idée, portée essentiellement par Brousseau mais aussi quelques autres, qu'on ne peut pas séparer de la pédagogique du Mathématique dans la réflexion sur l'enseignement des Mathématiques et qu'il est nécessaire d'étudier les phénomènes d'enseignement dans la complexité de la classe (PERRIN-GLORIAN; BELLEMAIN, 2016, p. 1).

Nous pouvons constater ci-dessus la place donnée à la compréhension du fonctionnement réel de la classe dans une école. C'est dans ce contexte particulier que la (DM) indique trois éléments fondamentaux qui jouent un rôle essentiel dans les phénomènes d'enseignement et d'apprentissage, à savoir: l'élève - l'enseignant (de mathématique) - le savoir (mathématique) (ARTIGUE, 1996).

Brousseau (1996) détermine le champ d'action et les intérêts de la (DM), lorsqu'il précise que la description suivante et la démarcation en tant que champ de recherche et le domaine d'intérêt, en particulier, avec une attention particulière portée aux processus cognitifs et aux instruments méthodologiques capables de fournir l'évolution de leurs apprentissages. Brousseau (1986) explique les intérêts de (DM) comme suit:

La didactique des Mathématiques étudie les activités didactiques, c'est-à-dire les activités qui ont pour objet l'enseignement, évidement dans ce qu'elles ont de spécifique aux mathématiques. Les résultats, dans ce domaine, sont de plus en plus nombreux, ils portent sur les comportements cognitifs des élèves, mais aussi sur les types de situations mis en œuvre pour les enseigner et sur tous les phénomènes auxquels la communication du savoir donne lieu. La production ou l'amélioration de moyens d'enseignement trouve dans ces résultats bien plus que des objectifs ou des moyens d'évaluation, elle y trouve un appui théorique, des explications, des moyens de prévision et l'analyse, des suggestions, voire des dispositifs et des méthodes (BROUSSEAU, 1996, p. 45).

En essayant de faire le lien entre la (DM) et la didactique professionnnelles (DP), nous dressons une brève présentation de certains éléments avec les hypothèses qui semblent permettre de relier ces deux 
champs d'étude. Nous considérons ici le travail de Pastré (1999) sur la (DP) a partir duquel nous tentons de faire une connexion théorique avec la (DM).

D'abord, nous soulignons que la (DM) se développe en prenant en compte l'épistémologie du savoir mathématique, qui est un moteur pour le développement des situations didactique (SD) qui considèrent l'importance de la transposition didactique (CHEVALLARD, 1991), ce qui tend à relier le travail du mathématicien professionnel aux situations proposées aux élèves qui, en général, sont construites par le chercheur et développées avec l'aide de l'enseignant dans la classe et à travers d'une séquence d'enseignement.

À ce moment, il est important de souligner que des mathématiciens professionnels, comme Dieudonné (1987), ont proposé des modifications del'enseignement des mathématiques en tenant compte des effets du mouvement de la Mathématique Moderne qui a eu une forte répercussion en France. Selon Dieudonné (1987), l'enseignement des Mathématiquesse développait sur la forme scientifique de la présentation des connaissances, connue sous le nom de présentation formaliste et axiomatique (DIEUDONNÉ, 1987), généralement construite et élaborée au sein de l'université. Sa proposition était que ce développement avait besoin de subir des modifications de façon à ce que les élèves et les enseignants pourraient participer d'un processus de personnalisation des connaissances, de façon à prendre en compte pour certains contenu l'ensemble de variables et de déterminants historiques, personnels et pragmatiques qui sont importants pour définir, délimiter et caractériser leurs approches individuelles.

Un des travaux développés à l'époque et qui constitueun des phénomènes particulièrement intéressants de la (DM) a été la transposition didactique (CHEVALLARD; 1991; 1996) ce qui implique une notion théorique d'intérêt centrée sur l'ensemble des transformations impliquant le savoir savant et le savoir à enseigner. Dans cette culture de recherche, la distinction et le rôle fonctionnel entre savoir et connaissance est très important. 
Arsac (1991) a développé une analyse du développement et de la généralisation de la notion de transposition didactique. L'auteur souligne deux points fondamentaux qui méritent plus d'attention: le problème de la légitimation du contenu de l'enseignement; l'apparition systématique d'un abîme entre savoir savant et savoir enseigné. Dans l'extrait suivant, Arsac (1991) explicite que la légitimation du savoir à enseigner ne peut pas être laissé aux seuls enseignants, elle doit être légitimé aussi par la société. De plus, le savoir à enseigner doit être original et en rapport avec le savoir savant, ce qui correspond à la transposition didactique au sens de Chevallard (1991).

La question de la légitimation relève de la remarque suivante: le choix d'un contenu d'enseignement ne relève pas seulement d'une initiative du maître, il doit apparaître comme légitime aux yeux de la société. [...] L’originalité du savoir enseigné par rapport au savoir savant, due aux contraintes qui pèsent sur lui, suppose un travail de fabrication du savoir enseigné a partir du savoir savant. C'est le travail de fabrication qui constitue par définition la transposition didactique (ARSAC, 1991, p. 39).

Ce sont les questions posées par les difficultés associées àla transposition didactique, en particulier, les questions de légitimation et d'originalité, qui ont conduit Chevallard (1991) à introduire les concepts fondamentaux de la didactique: perspectives apportées par une approche anthropologique. Pour cela, Chevallard (1991) explicite que la théorie anthropologique rompt avec le fantôme de la pureté etd'isolationnisme épistémologique, en se plaçant au sein de l'univers des savoirs. Elle demeure bien dans l'épistémologie, mais tout en représentant une partie de l'anthropologie. Cette considération lui permet de définir la didactique par rapport àl'anthropologie en soulignant que l'anthropologie correspond ici à l'étude de l'homme et de la société.

A partir de cette remarque, Chevallard (1992) défini la (DM) comme un sous-domaine de l'anthropologie des mathématiques, c'est-àdire, l'anthropologie didactique des mathématiques se consacre à l'étude 
de l'homme enseignant et apprenant des mathématiques. C'est a partir de cette définition que l'auteur introduit les éléments primitifs de la théorie et les notions de rapports personnels et institutionnels, ce qui le conduit à définir l'anthropologie de la connaissance ou l'anthropologie cognitive comme l'ensemble des rapports institutionnels et personnels avec les objets auxquels ils sont en rapport, et qu'ils font vivre, ainsi que leurs genèses et leurs changements.

En considérant que, selon Chevallard, la (DM) se consacre à l'étude de l'homme enseignant et apprenant, il nous semble important d'essayer de mieux comprendre les compétences professionnelles, en général, pragmatiquement construites et acquises, au fil des années de travail en tant qu'enseignant. Ces compétences acquises sont l'un des outils qui peuvent aider l'enseignant à transformer les savoirs à enseigner en savoirs enseignés. Ainsi, d'une certaine manière, nous pouvons considérer que le spectre des caractéristiques professionnelles attendues des enseignants, de la part du système éducatif, implique un champ de connaissances qui dépasse les limites de la seule connaissance mathématique (ALVES, 2017).

Ici, il nous semble important de rappeler, une autre notion fonctionnelle, préconisée par la théorie des situations didactiques (TSD), impliquant un modèle prédictif d'un ensemble de transformations du savoir, dans le contexte de l'enseignement (Brousseau, 1986). Pour cela, nous observons que certains phénomènes peuvent être enregistrés et analysés au moyen d'un modèle simplifié des rapports anticipés par les interactions des éléments du triangle didactique classique: enseignant élève - savoir. Dans ce contexte, la (TSD) propose quatre situations de modélisation dialectique pour la connaissance mathématique:

I. Situation dialectique d'action: l'éleve est confronte à une situation qui lui pose problème. Dans sa recherche de solution, il produit des actions qui peuvent aboutir à la création d'un savoirfaire. Il peut plus ou moins expliciter ou valider ses actions, mais la situation d'action ne l'exige pas. (DOUADY, 1984, p. 6). 
II. Situation dialectique de formulation: Des conditions différentes rendent nécessaires un échange des informations et lá création d'un langage pour assurer l'échange. Dans la situation de formulation, l'éleve peut justifier ses proposition, mais la situation ne l'exige pas. (DOUADY, 1984, p. 6).

III. Situation dialectique de validation: Les échanges ne concerne plus seulement les informations mais aussi les déclarations. Il faut prouver ce qu'on affirme autrement que par l'action. C'est l'objetif de la situation de validation. Ici, nous sommes mis en jeux deux aspects importants: la necessite de prouver est liée à la situation à laquelle on se trouve et la preuve est un acte social. (DOUADY, 1984, p. 6).

IV. Situation dialectique d'institutionnalisation: Ensemble de transformations qui font d'un savoir mathématique un patrimoine culturel de savoir mathématique pour un groupe d'élèves et qui doit être intégré. La fonction de chacune de ces situations dialectiques de connaissances impliqueun changement de perspective et d'activités développées à la fois par l'enseignant et par les élèves.

Dans ce contexte, Brousseau (1986, p. 62) affirme que "dans la conception la plus générale de l'enseignement, le savoir est une association entre les bonnes questions et les bonnes réponses. L'enseignant pose un problème que l'élève doit résoudre". Par rapport à l'affirmation qui précède, il est important de décrire une notion impliquant et indiquant les éléments principaux d'un système conçu par Brousseau (1986). En effet, l'auteur décrit un ensemble de règles et d'obligations attendues, tant par l'enseignant que par l'élève. Par exemple, à partir de la notion de dévolution, du choix de certains problèmes, on s'attend à ce que l'élève puisse accepter les conditions proposées par l'enseignant et, de ce fait, l'élève perçoit certaines intentions d'enseignement du maître. De plus, nous ne pouvons pas ignorer "l'adaptation à un milieu, c'est ce qui permet de produire des connaissances nouvelles, ce qui est modélisé 
en (TSD) par une situation a-didactique, c'est-à-dire un milieu comme système antagoniste de l'élève qui apporte des rétroactions à ses actions". (PERRIN-GLORIAN; BELLEMAIN, 2016, p. 13).

Cependant, nous ne pouvons pas ignorer certains obstacles qui se dressent, de façon imprévue, et qui peuvent déterminer un ensemble de difficultés à la compréhension de l'élève. En ce sens, nous rappelons quelques considérations de Bachelard (1934), qui montre l'importance $\mathrm{du}$ traitement de plusieurs situations particulières qui permettent de créer des images mentales avant de passer à une forme géométrique jusqu'àparvenir à la forme abstraite, comme nous pouvons le constater dans l'extrait qui suit.

Puisque tout savoir scientifique doit être à tout moment reconstruit, nos démonstrations épistémologiques auront tout à gagner à se développer au niveau des problèmes particuliers, sans souci de garder l'ordre historique. Nous ne devrons pas non plus hésiter à multiplier les exemples si nous voulons donner l'impression que, sur toutes les questions, pour tous les phénomènes, il faut passer d'abord de l'image à la forme géométrique, puis de la forme géométrique à la forme abstraite, poursuivre la voie psychologique normale de la pensée scientifique (BACHELARD, 1934, p. 10).

Il est important de remarquer ici que, à plusieurs reprises dans l'enseignement, c'est justement le contraire qui est développé et que la composante épistémique ne se prête pas à une description complète $\mathrm{du}$ fonctionnement de tous les obstacles cognitifs avant même l'acte d'apprendre. Ainsi, une composante considérée et intervenant dans le progrès de la science, fait référence à la composante cognitive ou psychologique du sujet, en fonction de leur capacité d'adaptation en situation d'action et de surmonter les obstacles. En effet, nous tirons une telle conclusion du fragment qui suit.

Quand on cherche les conditions psychologiques des progrès de la science, on arrive bientôt à cette conviction que c'est en termes 
d'obstacles qu'il faut poser le problème de la connaissance scientifique. Et il ne s'agit pas de considérer des obstacles externes, comme la complexité et la fugacité des phénomènes, ni d'incriminer la faiblesse des sens et de l'esprit humain: c'est dans l'acte même de connaître, intimement, qu'apparaissent, par une sorte de nécessité fonctionnelle, des lenteurs et des troubles. C'est là que nous montrerons des causes de stagnation et même de régression, c'est là que nous décèlerons des causes d'inertie que nous appellerons des obstacles épistémologiques. La connaissance du réel est une lumière qui projette toujours quelque part des ombres. Elle n'est jamais immédiate et pleine. Les révélations du réel sont toujours récurrentes (BACHELARD, 1934, p. 16).

Brousseau (1996) a manifestement fourni une expansion du point de vue bachelardien, dans la mesure où il décrit des obstacles de nature cognitive, des obstacles ontogénique, des obstacles méthodologique. Une telle catégorisation des obstacles découle naturellement d'une modélisation de l'activité de l'enseignant et de l'élève face à un ensemble fondamental de situations propres à un savoir donné, avalisé par l'enseignant. Ainsi, Brousseau (1996) développe une description de certains moments attendus, tant de la part de l'enseignant que de la part de l'élève, face aux situations didactique et a-didactiques et aux interactions avec les connaissances mathématiques. Effectivement, l'auteur explique et définit la nature d'une situation a-didactique, comme décrit dans l'extrait qui suit.

L'élève sait bien que le problème a été choisi pour lui faire acquérir une connaissance nouvelle mais il doit savoir aussi que cette connaissance est entièrement justifiée par la logique interne de la situation et qu'il peut la construire sans faire appel à des raisons didactiques. Non seulement il le peut, mais il le doit aussi car il n'aura véritablement acquis cette connaissance que lorsqu'il sera capable de la mettre en œuvre de lui-même dans des situations qu'il rencontrera en dehors de tout contexte d'enseignement et en l'absence de toute indication intentionnelle. Une telle situation est appelée situation a-didactique (BROUSSEAU, 1996, p. 16). 
Brousseau (1996, 2002) explique que la théorie prévoit d'être conscient que l'activité de l'élève est centrale, car elle sera pratiquement dissociée de la figure de l'enseignant et qu'elle a besoin d'une nouvelle posture de la part des élèves face aux nouvelles situations de recherche mathématique, car celles-ci nécessitent l'utilisation ou l'adaptation de ses connaissances mathématiques acquises dans les temps précédents. Ceci impliqué un large ensemble d'interactions du système éducatif, des problèmes particuliers et spécifiques qui conduisent à la notion de situation didactique (BROUSSEAU, 1996, 2002).

Ceci nous a conduit à identifier certains aspects ignorés ou qui peuvent être mieux décrits dans le domaine de la didactique des mathématiques. En effet, basé sur un fondement cognitiviste de la connaissance, Brousseau (1986) consacre une attention totale et unique au développement cognitif et intellectuel de l'élève. Mais, il nous paraît important aussi d'étudier le processus d'enseignement et d'apprentissage, les processus d'apprentissage personnels du professeur de mathématiques dans son environnement de travail, les changements nécessaires de son activité et de sa régulation constante au sein du système éducatif, compte tenu des divers phénomènes et notions qui, dans ce sens n'ont pas été traités par la (TSD), ce qui est compréhensible une fois que la (TDS) est centrée sur le développement des l'élèves. De cette manière, il est important d'envisager un cadre théorique explicatif pour l'activité du professeur de mathématiques.

En effet, Brousseau (1996) met en avant un ensemble d'attentes, impliquant la responsabilité de l'élève d'accepter des situations proposées par l'enseignant, ce qui rend l'enseignant un peu à part de la situation, où il ne fait que contrôler le travail développé pendant les séances, en laissant beaucoup de place aux élèves.

Cependant, il nous semble important d'insister sur un ensemble de tâches professionnelles et de problèmes qui peuvent apparaître au sein de l'institution de travail elle-même, le milieau d'exécution et de développement dans le lieu de travail de l'enseignant de mathématique. Pour développer son travail, l'enseignant sera confronté à des conditions 
et des contraintes imposées par le système éducatif, pour lesquelles, souvent, il n'a pas de moyens de trouver tout seul des solutions pour les résoudre. C'est pourquoi, nous décrivons ici la notion de dévolution de l'enseignant de mathématiques, dans le sens qu'il faut qu'il exécute un ensemble d'activités souhaitées par le système éducatif, mais qui ne sont pas nécessairement explicitées ou visiblement réglementées par des documents prescripteurs ou à travers les personnes responsables à différents niveaux de travail, en particulier ceux qui contrôlent le fonctionnement quotidien de l'école.

De plus, l'activité d'enseignant de mathématiques ne peut être objectivée à partir d'un point de vue des tâches préalablement défini, en négligeant le caractère qualitatif de ses choix, les modifications et les changements de comportement, face à des problèmes erratiques et imprévus rattachés aux tâches de développement du processus d'enseignement et d'apprentissage, problèmes découlant de l'exécution de tâches professionnelles elles-mêmes.

Dans la section suivante, nous abordons quelques notions de didactique professionnelle (DP) dans le but de fournir une perspective théorique un peu plus précise de ce qui paraît être un consensus sur le rôle de l'enseignant de mathématiques. Nous ne développerons certainement pas une discussion exhaustive des principales catégories théoriques de (DP).

\section{La didactique Professionnelle (DP)}

L'activité de l'enseignant, son système de régulation des activités, les tâches effectuées quotidiennement et les compétences attendues par son lieu de travail et de développement professionnel ne peuvent être compris uniquement, par le champ épistémique issu du savoir mathématique scientifique constitué, par exemple, comme objet de sa transposition didactique (CHEVALLARD, 1991). Pour cette raison, une compréhension globale est essentielle, provenant d'une analyse du travail de l'enseignant des mathématiques, vu comme un travailleur de 
l'enseignement d'une discipline spécifique ou d'un opérateur de l'éducation qui développe un ensemble d'activités spécialisées, prescrites et récurrentes, conditionnées par des concepts pragmatiques (MAYEN, 2012).

Ainsi, avant de poursuivre notre discussion, il semble nécessaire de comprendre l'objet des études de recherche et des dispositifs employés par la (DP), pour cela nous nous rapportons à la définition fondamentale de Pastré, Mayen \& Vergnaud (2006), qui suit.

Pour un certain nombre de ses créateurs, la Didactique Professionnelles est née au sein et dans le prolongement de la formation des adultes. Une des formes qui apparait à ce moment-là et qui peut être considérée comme l'invention la plus caractéristique de la formation professionnelle continue (FPC) est l'ingénierie de formation. C'est un champ de pratiques qui consiste à construire des dispositifs de formation correspondant à des besoins identifiés pour un public donné dans le cadre de son lieu de travail. La formation scolaire a tendance à décontextualiser les apprentissages. L’ingénierie de formation va insister au contraire sur le contexte social dans lequel doit s'effectuer l'apprentissage d'adultes en formation (PASTRÉ; MAYEN; VERGNAUD, 2006, p. 146).

Ici, il est important de remarquer que dans le travail de recherche, nous avons pu observer de façon récurrente, des réflexions sur les implications possibles de la (DM) dans le domaine des études pour la (DP). En effet, Pastré, Mayen \& Vergnaud (2006) introduisent, de façon claire et précise, un processus d'extension et de généralisation de la notion de transposition didactique qui, dans le cas de la (DM), est clairement mis en évidence par un champ épistémique précis. Cependant, dans le contexte de la (DP), on peut avoir besoin de la notion de compétence professionnelle de l'enseignant, afin de réguler la qualité de la transposition didactique (CHEVALLARD, 1991) d'un certain contenu précis des mathématiques, ainsi que la stabilisation d'un ensemble caractéristique des routines qui garantissent leur réplication dans les activités. 
L'idée de transposition recouvre deux idées distinctes. La première, très importantes chez Chevallard, est que le contenu de l'enseignement des Mathématiques résulte de deux processus de transformation: la transformation du savoir savant en savoir à enseigner, la transformation du savoir à enseigner en savoir effectivement enseigné. La seconde résulte d'une extension de sens du mot 'transposition', et d'un changement de sens par la même occasion: toute situation de référence, scientifique ou professionnelle, appelle des transformations lorsqu'on l'utilise comme situation d'enseignement de l'apprentissage: simplification, suppression de certaines variables, choix de cas prototypiques, etc. (PASTRÉ; MAYEN; VERGNAUD, 2006, p. 150).

Dans le contexte de la (DP), il est inévitable de prendre en considération une extension du rapport aux activités professionnelles et, surtout, de la signification des activités de l'enseignant de mathématiques, qu'il faut discerner comme professionnel ou travailleur, c'est-à-dire, les activités qui sont en rapport avec la profession doivent être considérées. De cette manière, il nous semble important de donner une attention particulière au fonctionnement des concepts pragmatiques, car selon la (DP), il faut prend en compte toutes les activités réalisées par les enseignants.

Pastré (2002, p. 12) a identifié certaines propriétés fondamentales des concepts pragmatiques dans de nombreux cas ou dans la plupart des circonstances de notre vie. Ainsi, selon lui, nous faisons nos choix et recourrons à des actions tacites, qui, selon Mayen (2012), sont sous forte influence de la dimension pragmatique, des situations quotidiennes et des situations professionnelles.

Pastré (2002, p. 12) explique que les concepts pragmatiques servent principalement à établir un diagnostic préliminaire des situations professionnelles, en vue de l'efficacité et de la réalisation des buts définis. Ce qui est vu, dans cette condition, n'est pas révélé par la composante épistémique ou formelle des connaissances scientifiques et techniques, mais par son biais pragmatique. Pastré (2002, p. 13) explique que "le diagnostic d'une situation ne peut être satisfait avec le rapprochement et la globalisation. Il est nécessaire de sélectionner ce 
qui est présenté dans la situation comme vraiment pertinent". Dans ce scénario, par conséquent, la distinction entre les novices (apprentis) et les professionnels expérimentés sera la caractéristique distinctive. Par exemple, les professionnels expérimentés extraient peu d'informations nécessaires sur la situation et s'en tiennent très souvent à certains détails qui peuvent être relativisés ou négligés par le stagiaire débutant.

Pastré (2002, p. 13) remarque que "un concept pragmatique est caractéristique d'une situation professionnelle, et en cela il se distingue des concepts quotidiens de Vygostki. Un concept pragmatique est spécifique à une classe de situations assez délimitées". Ici, il nous semble qu'il y a une certaine similarité entre (TSD) et (DP). Nous avons considéré d'abord une forte attention donnée à un ensemble de caractéristiques des situations qui servent à décrire, déterminer et caractériser une situation d'apprentissage d'une connaissance mathématique spécifique. De plus, pour une situation d'apprentissage, il y a un répertoire de compétences nécessaires pour la régulation et l'exécution d'une certaine tâche professionnelle. En effet, Mayen (2012) indique que la notion de situation occupe une place particulière dans la (DP), étant donné qu'elle apporte la réponse à certains problèmes pratiques et théoriques. Ainsi, nous ajoutons la distinction entre les termes suivants discutés par Mayen (2012): situation professionnelle, situation, situation didactique professionnelle. Afin d'expliquer ce qu'il entend par ses trois types spécifique de situations, l'auteur développe la description qui suit:

Penser les situations de travail à partir des perspectives ergonomiques et psychologiques permet de conserver une position à partir de laquelle la formation n'est pas inféodée aux exigences des situations professionnelles et à leurs exigences. L'analyse du travail pour la formation débouche dans bien des cas sur la mise en évidence des raisons pour lesquelles ce qui arrive dans cette situation ne relève pas (et parfois surtout pas) d'une perspective de formation. Comprendre suffisamment de choses aux situations de travail pour pouvoir parvenir à une telle conclusion constitue une raison déjà suffisante pour nous intéresser aux situations professionnelles. On peut constater que, dans bien des situations, la confusion d'esprit, les manifestations 
d'incompétence, les erreurs et les pratiques approximatives ou encore les obstacles à l'apprentissage, n'ont pas grand-chose à voir avec les connaissances et les capacités des personnes, mais relèvent de bien d'autres facteurs qui constituent les situations professionnelles, ou, d'autres termes, qui constituent les conditions mêmes du travail. La formation peut parfois ne rien avoir à y faire (MAYEN, 2012, p. 61).

Dans la citation ci-dessus, nous observons une possibilité évidente pour l'extension de la notion d'obstacles qui, dans le contexte de la théorie des situations didactiques, consacre toute son attention à l'élève, bien que du point de vue de la (DP) nous comprenions la fonction incontournable de certaines situations complexes, intrinsèquement liées à la nature de l'activité qui caractérise la profession. De l'union de ces deux préoccupations, nous nous engageons irréductiblement sur le terrain de l'apprentissage des adultes et leur formation professionnelle. En ce sens, Baudouin (1999, p. 150) observe que pour mieux comprendre l'activité employée dans le travail, il est important d'analyser les composantes éducatives de l'éducation des adultes. En ce sens, l'auteur souligne que:

Le modèle d'expertise se définit ainsi par un mouvement de bascule irréversible, dès lors que la logique des savoirs à elle seule ne suffit plus à le fonder, parce qu'il s'enrichit des logiques d'action qui trouvent leurs cours dans le champ pratiques, lesquelles disposent de leurs rationalités propre qu'il reste à honorer. L'expertise apparaît comme une combinaison de deux régimes de familiarité, à des savoirs et à des milieux d'activité. Les formation d'adulte sont confrontées en permanence dans leur intervention à cette double logique de l'expertise, celle des registres des savoirs et celle des registres de l'action au principe même de leur intervention et de ses finalités, et que recouvre à nos yeux le thème de la compétence (BAUDOUIN, 1999, p. 151).

Dans la section précédente, nous avons vu, d'une part, un modèle comparatif et la façon dont l'enseignement des mathématiques fonctionne, étant donné les multiples variables qui viennent de la classe. D’autre 
part, une compréhension du fonctionnement de l'activité et de l'action professionnelle de l'enseignant de mathématiques ne peut être simpliste.

En effet, il devient nécessaire de considérer la logique constitutive de la connaissance épistémique et scientifique construite dans un environnement académique de formation initiale. De plus, la logique de l'action, la logique interne des actions, l'exécution des tâches et l'économie d'effort humain dans l'exécution des tâches et des procédures internalisée et perfectionnée par l'enseignant expert, face à des problèmes professionnels complexes, doit être comprise dans le but de perfectionner les mécanismes de formation des adultes, visant l'enseignement. Dans la figure 1, nous comparons le système didactique introduit par Brousseau (1986).

Figure 1 - Le triangle didactique classique de l'enseignement des disciplines et, à droite, une figure pyramidale qui offre un cadre d'analyse pour la formation des professionnels

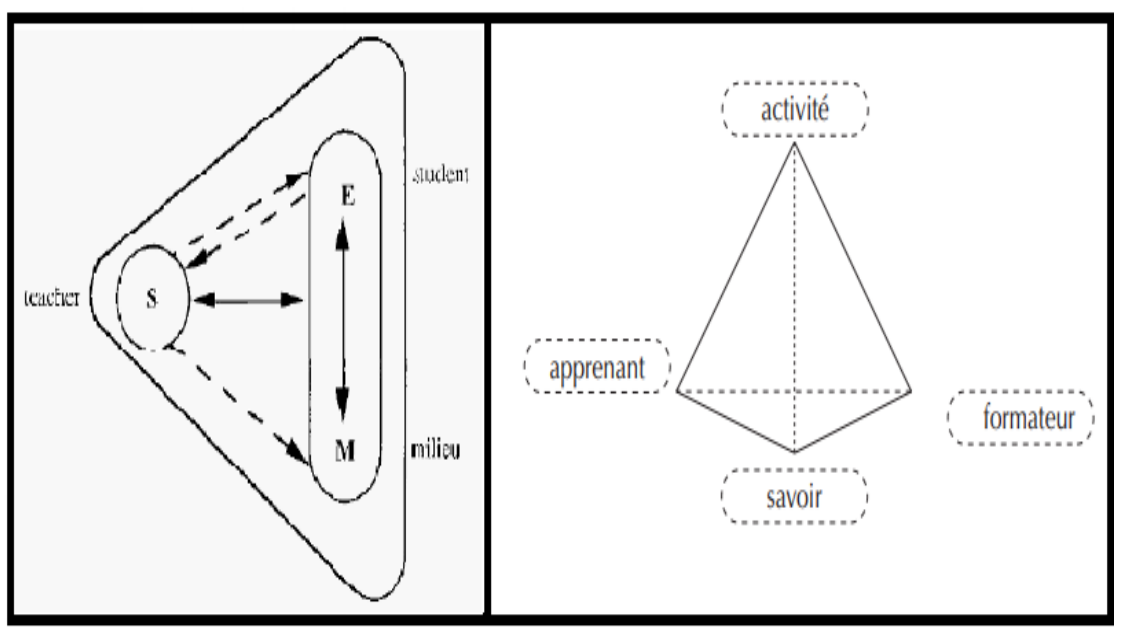

Source: Brousseau (2002) et Baudouin (1999).

Baudouin (1999) relève certaines limites concernant l'enseignement des disciplines classiques, étant donné que "l’orientation des disciplines affirme traditionnellement le rôle central du contenu de la connaissance et de la marque d'enseignement qui les concerne”. Enfin, 
Baudouin (1999, p. 152) relève certaines limites objectivables du triangle didactique classique, qui ne suffit plus à caractériser les conditions d'apprentissage propres à la formation des adultes et, par conséquent, des professionnels sur leur lieu de travail. Dans la figure 2 ci-dessous, nous observons le triangle didactique classique de l'enseignement des disciplines et, à droite, une figure pyramidale qui offre un cadre d'analyse pour la formation des professionnels, en indiquant les éléments essentiels: apprenant, activité, savoir, formateur.

À partir des éléments indiqués par Brousseau (1986) dans le contexte de (DM), nous défendons un caractère de non-suffisance afin d'expliquer de manière détaillée l'activité du professeur de mathématiques, les schémas cognitifs, les schémas mobilisés dans des situations de travail et apprendre au travail. Ainsi, dans la figure cidessous, nous présentons au lecteur une figure tridimensionnelle (3D) comportant une représentation informatique du modèle proposé par Baudouin (1999). De plus, nous pouvons visualiser deux plans bleu et rose. Le premier plan fait référence au plan didactique de mathématiques (DM) et le deuxième plan, en rose, au plan correspondant de la didactique professionnelle (DP).

Nous supposons ainsi une perspective d'analyse, de localisation du tétraèdre (3D) qui indique les éléments nécessaires à une évaluation approfondie de l'activité de l'enseignant, à la fois d'un point de vue éminemment conditionné par le champ épistémique disciplinaire. Dans ce cas, nous localisons le tétraèdre situé sur le plan de (DM). Et, dans le second cas, nous visons une perspective d'analyse de l'activité de l'enseignant, à partir d'um point de vue éminemment pragmatique. Dans le second cas, nous pouvons localiser la figure 3D sur le plan bleu de la (DP), la localisation du tétraèdre (3D). 
Figure 2 - Visualisation d'un modèle géométrique 3D significatif pour la formation des enseignants aux plans (DM) et (DP)

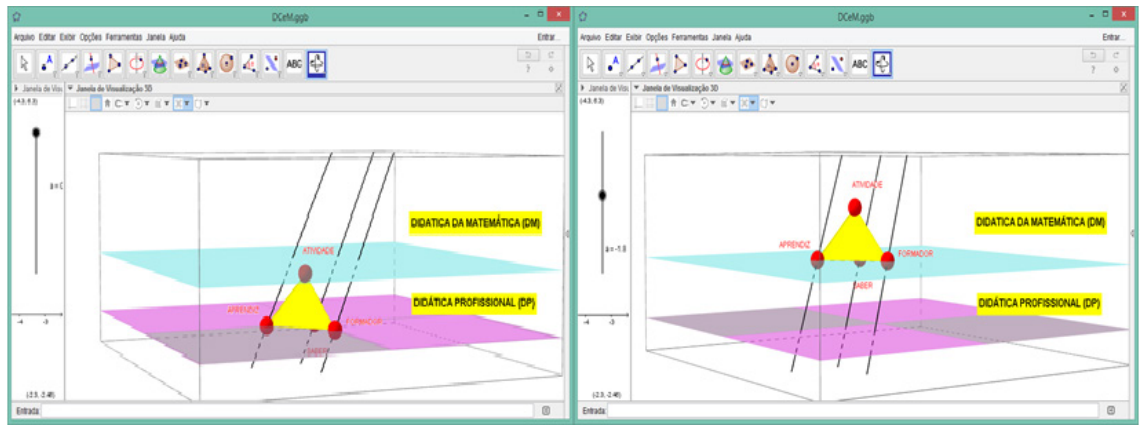

Source: Élaboration de l’auteur.

Le caractère dynamique de la représentation informatique cidessus permet de comprendre les déterminants disciplinaires de l'activité professionnelle. Pastré (2011) établit une distinction marquée entre le plan de la didactique des disciplines, nettement défini en termes de qualité de la transmission et l'acquisition des connaissances scientifiques et les intérêts de la didactique professionnelle (DP), lorsqu'il observe que:

Dela même manière, à son point de départ, la didactique professionnelle a tenu à marquer sa différence avec les didactiques des disciplines: celles-ci sont structurées autour de la transmission et de l'acquisition de savoirs. La didactique professionnelle se centre sur l'apprentissage d'activités. Or, on le verra, le fait de prendre comme objet non un savoir, mais une activité entraîne des conséquences importantes. On peut mentionner un dernier élément pour qualifier la didactique professionnelle: elle se donne comme objet le développement chez les adultes, avec cette idée forte que c'est dans le travail que la majorité des adultes rencontrent leur développement (PASTRÉ, 2011, p. 84).

Pastré (2011) présente une discussion sur la didactique professionnelle (DP) qui nous semble très proche de celle de Bachelard (1934), qui s'intéresse davantage à l'intérêt de comprendre la pensée 
scientifique, prenant comme exemples illustratifs l'apparition d'obstacles irréversibles et fonctionnels dans certains domaines disciplinaires, comme dans le cas des Mathématiques et de la Physique. Ici, dans le cas de la (DP), le scénario descriptif implique de faire face à des problèmes considérablement complexes et imprévus, bien que ces problèmes soient conditionnés par le poste de l'enseignant dans l'établissement ou le lieu de travail. En ce sens, Pastré (2011) décrit la notion importante de genèse conceptuelle.

Considérons maintenant les problèmes complexes. Il arrive qu'un acteur, doté d'un modèle opératif assez pertinent, s'attende à obtenir une «réponse» de la situation et se trouve désarçonné en constatant une contradiction entre la réponse qu'il attend et celle qu'il observe. Cela se produit généralement quand l'acteur se trouve confronté à une nouvelle classe de situations, par exemple un problème totalement nouveau, pour lesquels son modèle opératif, pertinent pour les situations qu'il a déjà rencontrées dans le passé, s'avère inadéquat dans le cas présent.

Cette contradiction peut être une occasion très intéressante d'apprentissage. Le modèle opératif de l'acteur n'est pas disqualifié pour autant: il est toujours valable pour traiter des classes de situations habituelles. Mais il demande à être élargi, réorganisé, pour être capable d'intégrer une nouvelle classe de situations professionnelles. On peut appeler "genèse conceptuelle» cet élargissement du modèle opératif de l'acteur, qui est aussi un approfondissement. Il ne s'agit pas d'ajouter de nouvelles connaissances aux connaissances anciennes. Il s'agit d'une restructuration des ressources cognitives du sujet, dans une perspective qu'on peut qualifier de bachelardienne (PASTRÉ, 2011, p. 92).

De cette manière, nous identifions des similitudes incontestables, concernant l'intérêt de l'élève pour l'apprentissage et, aussi, le professionnel lui-même, face aux situations et aux caractéristiques fondamentales de sa profession et travail. Nous pouvons maintenant parler de la notion d'obstacles épistémologiques ainsi que de la notion que nous défendons ici des obstacles professionnels. Le processus consistant à surmonter ces obstacles permettra de démarquer et de distinguer un 
enseignant inexpérimenté, novice, d'un enseignant expérimenté et surtout professionnellement compétent.

D'autre part, le sens que nous cherchons à souligner, lorsque nous parlons de la notion d'obstacles professionnels, nous nous référons à un ensemble de situations typiques et caractéristiques de chaque profession et que, conditionnés essentiellement par un ensemble de situations professionnelles, de tels obstacles se rencontrent pour une évaluation de la compétence professionnelle de l'enseignant, afin de gérer et de surmonter.

\section{Une perspective de complémentarité théorique}

Comme nous en avons discuté dans les sections précédentes, la (TSD) fournit un moyen d'analyse de l'activité de l'enseignant de mathématiques, principalement à la compréhension des variables autour des éléments circonscrits à l'environnement de la classe. À partir de cela, et des hypothèses de la (DP), nous soulignons une possibilité de généralisation et de corrélation immédiate impliquant certaines hypothèses communes et complémentaires. Pour ce biais d'analyse, nous rappelons l'adoption de certaines hypothèses d'origine piagétienne qui s'appliquaient à l'origine à l'environnement d'apprentissage des enfants (PASTRÉ; MAYEN; VERGNAUD, 2006), mais dans le cas du domaine de travail, nous envisageons une application au Brésil au scénario d'apprentissage des enseignants en formation initiale ou même des adultes dans leur propre lieu de travail, à savoir : des écoles brésiliennes, en se concentrant sur l'enseignement des mathématiques et dans certains cas, l'enseignement des Sciences.

Comme nous avons une formation liée à la (DM), cela nous conduit à être influencé par la culture et la tradition françaises et, nousa amené à choisir la (DM) et la (DP), qui sont deux domaines de recherche originaires de France. Pour mieux comprendre les interactions possibles entre ces deux domaines théoriques nous croyons qu'il est important de distinguer certains termes ou expressions qui, utilisés à l'origine dans 
la culture française, peuvent parfois présenter des difficultés lors d'une comparaison entre les phénomènes brésilien et français.

Par exemple, dans notre recherche au Brésil, nous proposons des études de cas avec des groupes d'enseignants, mais le développement de ces recherches sont différentes de ce qui est proposé par Pastré (2011), parce que nous considérons, plus particulièrement, les activités de l'enseignant selon le plan des tâches, selon le plan du poste de travail et, finalement, selon le plan de la profession. Mais, en fonction de la proposition de l'introduction des nouvelles technologies aux enseignants de la formation initiale, nous prenons en compte l'importance de la visualisation et des connexions des différents plans hiérarchiques de réflexion et d'insertion de l'action de l'enseignant de mathématiques.

Par exemple, dans la figure 3 , nous visualisons un triangle dynamique épistémique 2D, effectué à partir d'une construction réalisée avec le logiciel GeoGebra. Les sommets représentent l'enseignant-l'élèvele savoir. Nous distinguons d'abord un plan (en couleur verte) des tâches caractéristiques, développé avec plus d'intérêt et de répercussion en classe. Sur le côté gauche, nous observons un triangle dynamique (de couleur jaune), où nous identifions les notions fondamentales pour la didactique de mathématiques (DM): transposition didactique, obstacle epistemologique, compétence professionnelles.

Nous avons précédemment observé que des obstacles épistémologiques surviennent dans le contexte de la préoccupation pour la transposition didactique (CHEVALLARD, 1991) de disciplines spécifiques. De plus, en ce qui concerne l'apprentissage des élèves, leur évolution dans l'apprentissage des mathématiques sera conditionnée par le dépassement des obstacles représentatifs et la maîtrise de problèmes non complexes, dans un domaine éminemment épistémique. De même, dans le processus de transformation d'un enseignant débutant, c'est-à-dire un débutant dans le domaine de la familiarisation avec les tâches et situations professionnelles propres à sa profession, qui est en train d'acquérir des compétences nouvelles et de l'expérience, il convient del'inclure dans le plan correspondant des tâches primordiales par l'école. 
Figure 3 - Application d'une perspective de complémentarité de (DP) et (TSD) au plan des tâches de l'enseignant

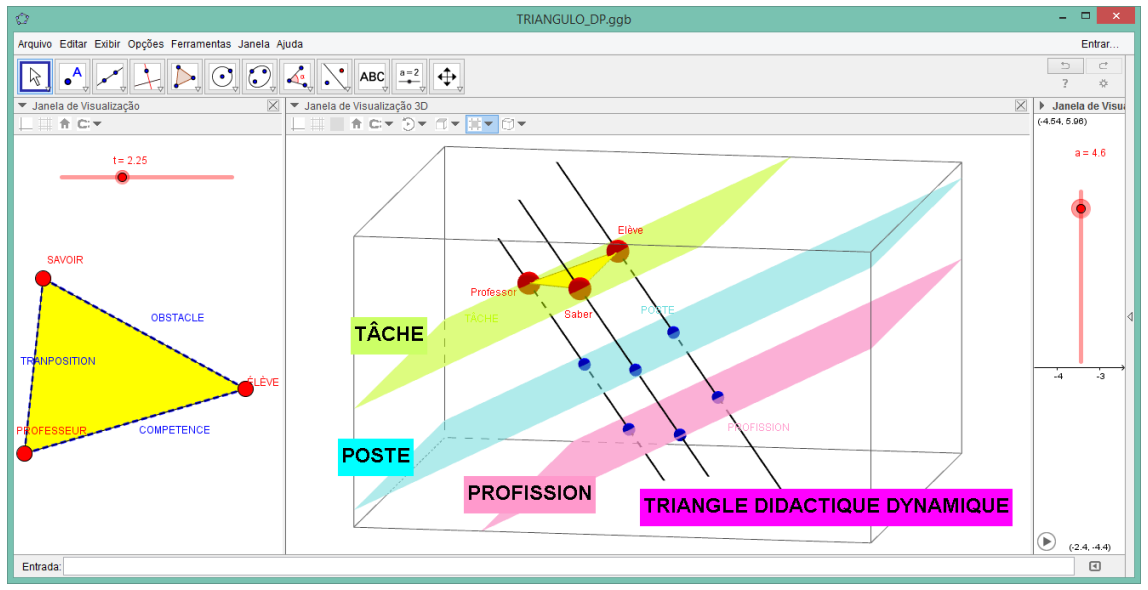

Source: Élaboration de l'auteur.

De cette manière, sur la figure 3 , nous mettons en évidence le plan du poste de travail. Dans ce scénario, nous rappelons les explications de Pastré (2011, p. 90) lorsqu'il considère que "le modèle opératif d'un acteur contient trois sortes d'organisateurs de l'activité: l'un se réfère à la situation de travail; un autre se réfère au groupe professionnel auquel se rattache l'acteur; le dernier constitue sa signature et dépend de son expérience passée". La proposition de Pastré (2011) s'exprime dans une signification immédiate pour notre cas d'interprétation du rôle de l'enseignant de mathématiques. En effet, dans le plan du poste de travail (le plan bleu de la figure 4), nous envisageons le développement d'un ensemble de concepts pragmatiques (MAYEN, 2012), essentiellement partagés par leurs collègues, qui effectuent des tâches hiérarchiques et distinguées dans un établissement d'enseignement donné. À aucun moment, sous l'influence de la pensée de Pastré (2011), nous ne négligeons une dimension pragmatique impliquant la notion de compétence professionnelle. 
Figure 4 - Application d'une perspective de complémentarité de (DP) et (TSD) au plan du poste de travail de l'enseignant

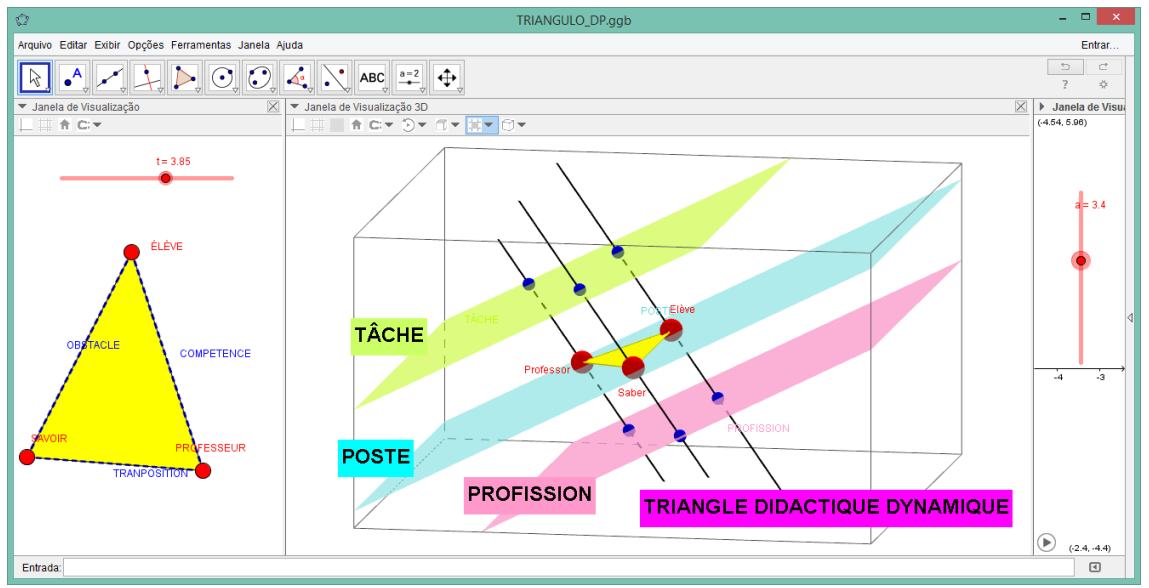

Source: Élaboration de l'auteur.

Ainsi, si nous nous référons à Brousseau (1996), nous savons que l'épistémologie de l'enseignant est déterminée par une méthode caractéristique de la connaissance mathématique et qu'elle constitue irrémédiablement un genre propre des langues, des gestes et du style d'activités mobilisé par un groupe, en fonction de certains postes de travail.

Au-delà de ce qui a été proposé par Brousseau, nous soulignons le besoin de la notion de la "transposition professionnelle" impliquant la transmission de connaissances professionnelles détaillées, avec une appréciation du processus et du rôle des novices et des professionnels expérimentés, notamment par le développement d'un style particulier ou d'un genre de langage, de gestes et d'informations intrinsèques au poste de travail. De plus, contrairement au type d'analyse employé par Chevallard (1991), dans le domaine professionnel, il est évident l'importance de la compétence professionnelle, qui correspond à la connaissance du sujet par rapport à ce que nous appelons «savoir mort», c'est-à-dire des motifs qui contribuent au processus de substitution et de disparition de 
certaines connaissances mathématiques et procédures correspondantes pour leur enseignement.

Figure 5 - Application d'une perspective de complémentarité de (DP) et (TSD) au plan d'exercice de la profession dans le système éducatif

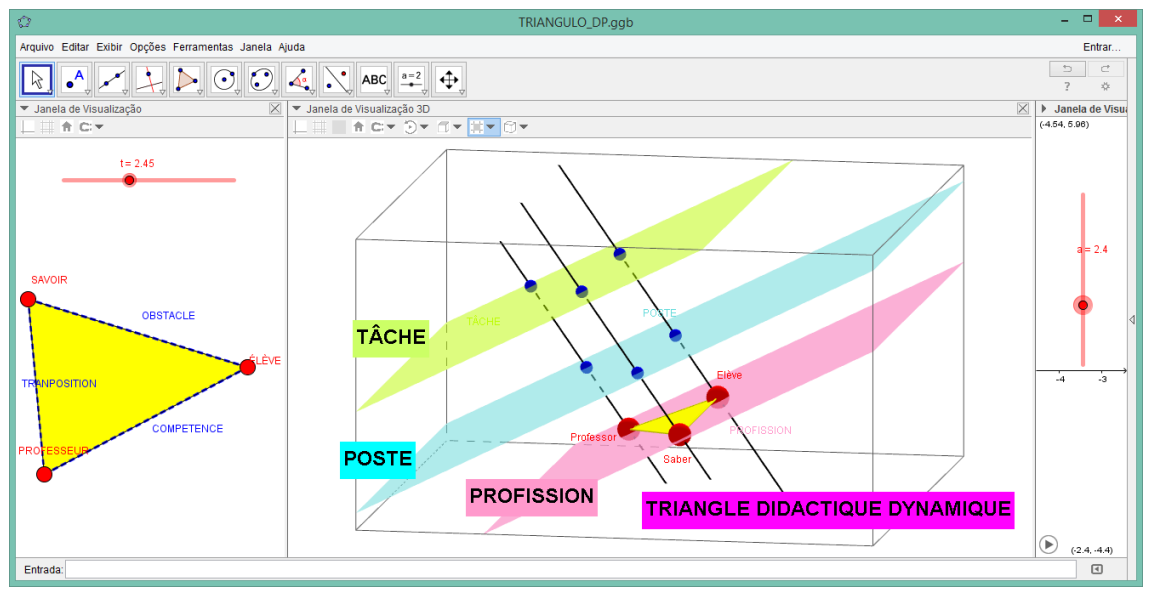

Source: Élaboration de l'auteur.

À partir de la remarque ci-dessus, il nous semble important de considérer la contribution de Clot \& Faita (2000), qui fournissent une description importante impliquant un ensemble d'habitus et de styles caractéristiques de la profession d'enseignant. En effet, au Brésil, un langage caractéristique définit parfois la forme et le pragmatisme de la pensée de l'enseignant de mathématiques.

Un ensemble de règles implicites fonctionne comme une sorte de "contrat professionnel" qui lie les personnes de la même profession et du même métier et qui détermine un ensemble de règles et de lois qui doivent être respectées par les membres du même groupe professionnel. Clot \& Faita (2000) expliquent les fonctions et les manifestations de ce que nous appelons le genre professionnel. 
Ces genres fixent, dans un milieu donné, le régime social de fonctionnement de la langue. Il s'agit d'un stock d'énoncés attendus, prototypes des manières de dire ou de ne pas dire dans un espacetemps sócio discursif [...] Ces énoncés retiennent la mémoire impersonnelle d'un milieu social dans lequel ils font autorité, donnent le ton. Ils trahissent les sous-entendus qui règlent les rapports aux objets et entre les personnes, traditions acquises qui s'expriment et se préservent sous l'enveloppe des mots. Ils prémunissent le sujet contre un usage déplacé des signes dans une situation donnée. Un genre est toujours attaché à une situation dans le monde social (CLOT; FAITA, 2000, p. 10).

Après la présentation des éléments des recherches qui nous ont déjà proposer le modèle complémentaire ci-dessus, nous présentons un tableau introductif qui établit un raisonnement de corrélation et de complémentarité entre certaines notions qui nous semblent généralisables. Il est important de remarquer que certaines d'entre elles n'ont pas été détaillées dans le domaine de la (DP), notamment ce qui a trait aux connaissances disciplinaires. Par rapport à l'observation ci-dessus, nous avons souligné les notions de: dévolution de l'élève, dévolution del'enseignant, situation didactique, situation professionnelle, situation didactique professionnelle, obstacle épistémologique, obstacle professionnel, transposition didactique, transposition professionnelle.

De toute évidence, nous n'assumons pas une position définitive, dans la mesure où, au Brésil, nous travaillons dans un cadre, dans lequel nous développons et nous mettons en œuvre deux théories, à travers la discussion pendant le travail, réalisée de façon empirique et encore en développement.

Ainsi, certaines des définitions ci-dessous peuvent être améliorées naturellement, à partir de nouveaux phénomènes dans lesquels sont employées la (TSD) et la (DP) dans la perspective de la formation des enseignants. 
Tableau 1 - Tableau comparatif des notions impliquant la (DM), (TSD) et (DP)

\begin{tabular}{|c|c|c|}
\hline Notions & $\begin{array}{c}\text { Didactique de mathéma- } \\
\text { tiques (DM) }\end{array}$ & $\begin{array}{l}\text { Didactique professionnelles } \\
\text { (DP) }\end{array}$ \\
\hline $\begin{array}{c}\text { Dévolution } \\
\text { Didactique } \\
\text { et } \\
\text { Professionnelles }\end{array}$ & $\begin{array}{l}\text { Ensemble de rapports et } \\
\text { d'activités de l'élève face aux } \\
\text { situations caractéristiques et } \\
\text { fondamentales proposées par } \\
\text { l'enseignant de mathématiques. }\end{array}$ & $\begin{array}{l}\text { Ensemble de rapports, d'activités } \\
\text { de responsabilité et de routines } \\
\text { développées par l'enseignant, dans } \\
\text { son poste de travail, attendu par } \\
\text { son institution de travail. Règles } \\
\text { déodontiques communes au groupe } \\
\text { d'enseignants. }\end{array}$ \\
\hline $\begin{array}{c}\text { Situation } \\
\text { Didactique } \\
\text { et } \\
\text { Professionnelles }\end{array}$ & $\begin{array}{l}\text { Situation didactique: Les } \\
\text { situations doivent tenir compte } \\
\text { à la fois de l'organisation des } \\
\text { mathématiques, des possibilités } \\
\text { d'apprentissage des élèves et } \\
\text { des conditions d'enseignement } \\
\text { des professeurs. } \\
\text { Cette notion a été introduite } \\
\text { à l'origine pour les } \\
\text { mathématiques par Brousseau } \\
\text { (1986). }\end{array}$ & $\begin{array}{l}\text { Situation didactique professionnelle: } \\
\text { Situations professionnelles } \\
\text { organisées autour d'une tâche } \\
\text { professionnelle et de l'apprentissage } \\
\text { qui en découle, déterminées par un } \\
\text { ensemble de prescriptions propres } \\
\text { à un poste de travail. Ensemble de } \\
\text { tâches visant un contexte ou un } \\
\text { scénario visant la formation planifiée } \\
\text { et l'acquisition de compétences } \\
\text { professionnelles. }\end{array}$ \\
\hline $\begin{array}{c}\text { Obstacle } \\
\text { Épistémologique } \\
\text { et } \\
\text { Professionnelles }\end{array}$ & $\begin{array}{l}\text { Obstacle épistémologique: } \\
\text { Obstacle, difficulté naturelle } \\
\text { et intrinsèque de résistance } \\
\text { contraire à l'acte même de } \\
\text { connaître une connaissance } \\
\text { scientifique et théorique. }\end{array}$ & $\begin{array}{l}\text { Obstacle professionnel: Situations } \\
\text { complexes, inattendues, avec des } \\
\text { caractéristiques d'une profession } \\
\text { particulière et même récurrentes } \\
\text { lors de l'exécution de certaines } \\
\text { tâches professionnelles en situation } \\
\text { de travail. }\end{array}$ \\
\hline $\begin{array}{c}\text { Transposition } \\
\text { Didactique } \\
\text { et } \\
\text { Professionnelles }\end{array}$ & $\begin{array}{l}\text { Transposition didactique: } \\
\text { Ensemble de transformations, } \\
\text { modifications et changements } \\
\text { nécessaires pour présenter une } \\
\text { connaissance mathématique, de } \\
\text { l'environnement académique au } \\
\text { contexte scolaire. } \\
\text { Initialement introduit } \\
\text { pour laDidactique des } \\
\text { Mathématiques par Chevallard } \\
\text { (1991). }\end{array}$ & $\begin{array}{l}\text { Transposition professionnelle: } \\
\text { Ensemble de phénomènes liés à } \\
\text { la transmission de connaissances } \\
\text { professionnelles impliquant des } \\
\text { professionnels inexpérimentés et } \\
\text { expérimentés ou, sinon, impliquant } \\
\text { des formateurs d'enseignants } \\
\text { professionnels visant à la } \\
\text { transmission des connaissances } \\
\text { aux futurs enseignants de } \\
\text { Mathématiques. }\end{array}$ \\
\hline
\end{tabular}

Source: Organisé par l'auteur. 


\section{Conclusion}

Dans les sections précédentes, nous avons discuter quelques éléments et notions représentatifs de la (DP) et de la (DM), impliquant une perspective théorique de recherche en développement au Brésil, afin d'améliorer les rapports objectifs et structurels de la formation académique des enseignants de mathématiques, dans les modalités initiales et continues. En particulier, nous soulignons une appréciation de certaines notions issues de la théorie des situations didactiques (TSD) qui permettent une sorte de modélisation et de théorisation des principaux éléments impliqués dans le contexte de l'enseignement et de l'apprentissage des mathématiques. Comme nous l'avons mentionné, l'influence des études développées au Brésil, avec la contribution théorique du domaine d'étude de la didactique des mathématiques, a déjà presque trente ans de répercussion. Cependant, malgré son apparition en France dans les années 1990, la didactique professionnelle n'a toujours pas encore d'application expressive et systématique au Brésil (ALVES, 2018a; 2018b; 2018c; 2018d).

De cette manière, nous adoptons une perspective de complémentarité théorique, impliquant les hypothèses de la (DM) et de la (DP) considérant que la (DM) fournit une riche interprétation des phénomènes intimement liés et conditionnés par le champ mathématique épistémique. Et, nous sommes aussi conduits à considérer les intérêts déclarés par la (DP) pour fournir une analyse théorique et psychologique du travail, dans la mesure où elle prend en compte les processus d'apprentissage acquis et nécessaires aux adultes et, plus particulièrement, aux enseignants, afin de développer progressivement leurs compétences professionnelles dans la durée, et compte tenu de la nécessité de développer des activités spécialisées et d'accomplir des tâches professionnelles.

De cette façon, après un certain temps, nous remarquons l'existence d'un processus de refondation et du besoin de rediscuter certaines notions issues de la (DM), comme par exemple l'Ingénierie 
Didactique (ID) (PERRIN-GLORIAN; BELLEMAIN, 2016), largement connue au Brésil. Il est important de remarquer que ce processus de refondation de l'Ingénierie Didactique (ID) est déjà en cours au Brésil et il y a des ingénieries du type parcours d'etude et de recherche (PER) développées par des étudiants de "doctorat ».

Par contre, comme nous le soulignons tout au long du travail, certaines considérations et hypothèses de Pastré (1999) ont besoin d'une plus grande appréciation systématique et théorique de la part des chercheurs brésiliens, surtout lorsqu'elle se réfère et cherche à extraire quelques implications de certaines notions classiques de la (DM), comme nous le proposons dans le tableau I ci-dessus.

Il est aussi important de souligner que la compétence professionnelle d'un individu se manifeste surtout face à des situations exceptionnelles, face à des tâches qui impliquent des caractéristiques originales et avec un certain contenu de nouveauté, face à des situations intrinsèquement complexes et pas nécessairement routinières, ceci a conduit à l'affirmation: "l'une des préoccupations de la didactique professionnelle (DP) n'est pas de rester dans l'analyse des situations de travail, mais d'analyser l'activité dans son développement et sa durée" (PASTRÉ, 2007, p. 26).

Enfin, nous rejoignons la contribution de Leclercq (2002) qui envisage un champ d'investigation précurseur abordant les phénomènes abyssaux entre les références de la formation en France des enseignants en formation initiale ou continue et les références et les besoins de la profession, sous réserve d'un ensemble de demandes de professionnalisme qui changent, ayant une vitesse plus rapide que l'élément constitutif et définissant des références de la formation et de l'ingénierie sociale (LECLERCQ, 2002). Dans la figure 6, nous observons un schéma capable de communiquer au lecteur certaines idées qui se sont répandues en Europe, et particulièrement en France, dans l'aprèsguerre et qui ont renforcé la notion d'ingénierie ou d'ingénierie de le développement professionnel du travailleur (ingénierie pédagogique, ingénierie de formation et ingénierie sociale). 
Dans notre cas d'étude, nous avons cherché à mettre l'accent sur une perspective d'accumulation d'œuvres et de dispositifs visant à perfectionner et à comprendre les mécanismes de la professionnalisation, c'est-à-dire un intérêt explcite pour l'ingénierie de formation didactique (EDF) (ALVES; ALVES DIAS, 2017).

Figure 6 - Leclerqc (2002) décrit les activités développées sur les pentes d'ingénierie développées en Europe

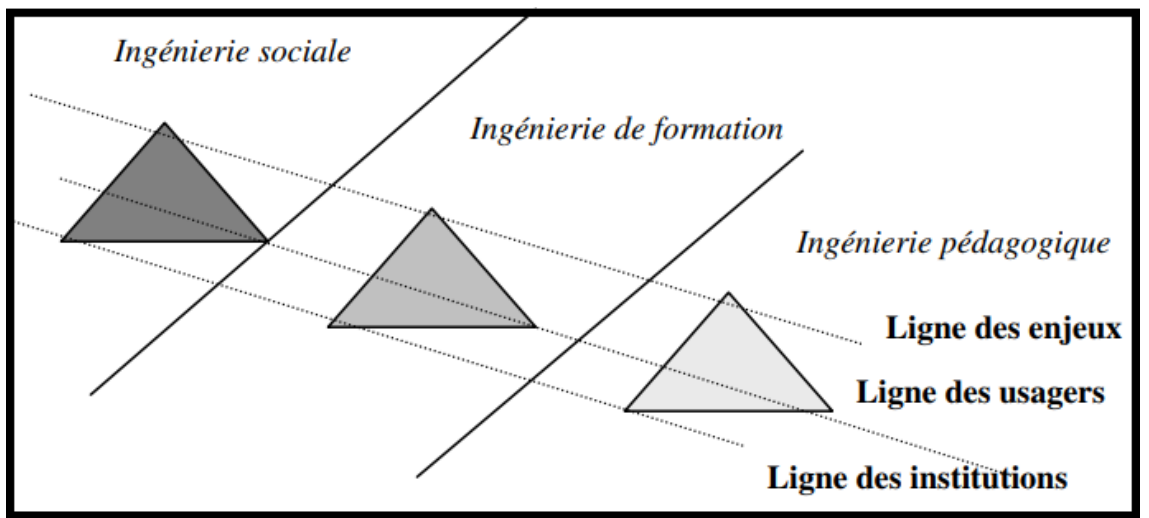

Source: Leclerque (2002).

Finalement, nous rejoignons Pastré (1999, p. 123) lorsqu'il avertit que "si nous voulons avancer sur les problèmes de compétences, nous devrons investir de nouveau dans les problèmes de contenu de la formation". Autrement dit, on en déduit, surtout lorsque l'on considère le scénario brésilien, que malgré une profusion d'actions parfois académiques et locales, en inversant les indicateurs inquiétants liés à la formation des professionnels de l'enseignement, certaines obsolescences et anachronismes restent intacts. 


\section{Références}

ALVES, F. R. V. Didactique des mathématiques: ses hypothèses d'ordre épistémologique, méthodologique et cognitif. Revista Interfaces da Educação, v. 8, n. 22, p. 131-150, 2016.

ALVES, F. R. V. Didactique des sciences et des mathématiques (DCeM): augmentation et implications de la formation des enseignants. Revist sobre $o$ ensino de ciências, v. 22, n. 3. p. 291-320, 2017.

ALVES, F. R. V. Didactique Professionnelle (DP) et la Théorie des Situations Didactiques (TSD): le cas de la notion d'obstacle et l'activité de professeur. EM TEIA: Revista de educação matemática e tecnológica ibero-americana, v. 9, n. 2, p. 1-25, 2018a.

ALVES, F. R. V. The professional didactics (PD) and didactics of sciences (DS) in Brazil: some implications for the professionalization of the science teacher. Acta Didactica Naposcencia, v. 11, n. 2, p. 105-120, 2018b.

ALVES, F. R. V. Mestrado (acadêmico) em ensino de ciências e matemática: a proposta do Instituto Federal do Ceará (IFCE). Revista Contexto \& Educação, v. 33, n. 105, p. 305-340, 2018c.

ALVES, F. R. V. Didactique Professionnelle (DP) et la Théorie des Situations Didactiques (TSD): le cas de la notion d'obstacle et l'activité du professeu. EM TEIA: Revista de Educação Matemática Iberoamericana, v. 9, n. 3, p. 1-25, 2018 d.

ALVES, F. R. V.; ALVES DIAS, M. Formação de Professores de Matemática: um contributo da Engenharia Didática. REVEMAT, v. 12, n. 2, p. 192-209, out. 2017. ARSAC, G. Un exemple d'évolution d'une théorie didactique : la transposition didactiques. Publication de l'Institute de Recherche mathématiques de Rennes, n. S6, p. 39-43, 1991.

ARTIGUE, M. Ingénierie Didactiques. In: BRUN, J. (org.). Didactique des mathématiques. Paris: Délachaux et Niestlé, 1996. p. 243-264. 
BACHELARD, G. La formation de l'esprit scientifique. 5. ed. Paris: Librairie philosophique J. Vrin, 1934.

BAUDOUIN, J. M. La competénce et le théme de l'acitivité: vers une nouvelle conceptualization didactique de la formation. Raison educative, v. 2, n. 2, p. 149168, 1999.

BROUSSEAU, G. Théorisation des phénomènes d'enseignement des mathématiques. Thése (Doctorat) — Université Bourdeaux I, Bourdeaux, 1986.

BROUSSEAU, G. Fondements et méthodes de la didactique des mathématiques. In: BRUN, J. Didactique des mathématiques. Paris: Délachaux et Niestlé, 1996. p. 45-110.

BROUSSEAU, G. Theory of didactical situations in mathematics didactique des mathématiques, 1970-1990. New York: Academic Publishers Editors, 2002.

CHEVALLARD, Y. La transposition didactique: du savoir savant au savoir enseigné. 2. ed. Grenoble: La pensée sauvage, 1991.

CHEVALLARD, Y. Concepts fondamentaux de la didactique: perspective apportées par une approche antropologique. In: BRUN, J. Didactique des mathématiques. Paris: Délachaux et Niestlé, 1996. p. 145-186.

CLOT, Y.; FAITA, D. Genres et styles en analyse du travail: concept et méthodes. Revue Theme, v. 4, n. 4, p. 7-42, 2000.

DIEUDONNÉ, J. Pour l'honneur de l'esprit humain: les mathématiques aujourd'hui. Paris: Hachette, 1987.

DOUADY, R. De la didactique des Mathématiques à l'heure actuelle. Le Cahier Rouge. v. 6, $\mathrm{n}^{\mathrm{o}} 1,1-40,1984$.

LECLERCQ, G. Quelques usages de l'activité d'ingénierie de formation. Revue Savoirs, v. 2, n. 2, p. 71-104, 2002.

MAYEN, P. Les situations professionnelles: un point de vue de didactique professionnelle. Revue Phronesis, v. 1, n. 1, p. 59-67, 2012. 
PASTRÉ, P. La conceptualisation dans l'action: bilan et nouvelles perspectives. Éducation permanente, v. 139, n. 1, p. 13-35, 1999.

PASTRÉ, P. L'analyse du travail en Didactique professionnelle. Revue Française de Pédagogie, v. 3, n. 138, p. 9-17, 2002.

PASTRÉ, P. La didactique professionnelle. In: Le Web TV formation professionnelles. Paris: AFPA, 2007. 1 filme (54min), sonoro, color.

PASTRÉ, P. La Didactique Professionelle. Education, Sciences \& Society, v. 2, n. 1, p. 83-95, 2011.

PASTRÉ, P; MAYEN, P.; VERGNAUD, G. La didactique professionnelles. Revue française de pédagogie, v. 3, n. 154, p. 145-198, 2006.

PERRIN-GLORIAN, M. J.; BELLEMAIN, P. M. L'Ingénierie didactique entre recherche et ressource pour l'enseignement et la formation des maitres. In: SYMPOSIUM DE DIDACTIQUE DES MATHÉMATIQUES DE L'AMÉRIQUE LATINE, 1., 2016, Bonito. Annales... Bonito: SBEM, 2016. p. 1-35.

Reçu: 22/05/2018

Recebido: 22/05/2018

Received: 05/22/2018

Approuvé: 22/10/2018

Aprovado: 22/10/2018

Approved: 10/22/2018 